\title{
Morenos Erbe im Coaching
}

\section{Astrid Schreyögg}

Dieses Heft ist einmal mehr eine Liebeserklärung der Herausgeber an Moreno. Schon in früheren Heften dieser Zeitschrift (4/1997 und 2/2005) haben wir schwerpunktmäßig auf die große Bedeutung Morenos für berufliche Beratungsformate aufmerksam gemacht. Auch in weiteren Heften, etwa in Arbeiten von Witte (3/2001 und 2/2007) oder in etlichen Beiträgen von Ferdinand Buer bestand jeweils ein Bezug zu Morenos Konzepten. Daneben haben wir auch immer wieder Beiträge publiziert, in denen nur indirekt auf das Moreno'sche Erbe rekurriert wurde, etwa mit Aufsätzen zur Imagination, zu Varianten des Rollenspiels oder zum ,inneren Team“. Denn auch diese methodischen Zugänge verweisen auf Moreno.

Methodische Aspekte des Moreno'schen Werkes erfreuen sich auch großer Beliebtheit in Tool-Büchern, in den „Supervisions-Tools“, die Neumann-Wirsig (2009) herausgegeben hat, oder in den von Rauen (2004, 2007) herausgegebenen Bänden mit Coaching-Tools, die bis heute in vielfachen Auflagen erschienen sind. Dabei ist allerdings einzuwenden, dass sich die meisten Autoren in den Tool-Büchern selten explizit auf Moreno berufen. Wer aber mit einschlägigen Ansätzen vertraut ist, erkennt schnell, dass Morenos Arbeiten Pate standen für diese oder jene Technik und für dieses oder jenes Arrangement. In den letzten Jahren erhielt Moreno für das Coaching und dabei besonders für das Life-Coaching noch einmal umfassende Bedeutung (Buer und Schmidt-Lellek 2008; Schmidt-Lellek und Buer 2011). In diesem Rahmen, in dem es um „Sinn, Glück und Verantwortung in der Arbeit" geht, erhält das Erbe Morenos einen sehr prominenten Stellenwert, denn hier geht es ja nicht so sehr um seine methodische Hinterlassenschaft, sondern mehr um seine Ethik und seine Anthropologie. Diese metamodell-theoretischen Aspekte begründen auch die große Beliebtheit des Moreno'schen Oeuvres in der Psychotherapie, in der Pädagogik und in verschiedenen Formaten beruflicher Beratung wie Supervision, Coaching und Organisationsberatung.

Online publiziert: 05.04 .2012

(C) VS Verlag für Sozialwissenschaften 2012

Dr. A. Schreyögg $(\bowtie)$

Breisgauer Str. 29, 14129 Berlin, Deutschland

E-Mail: info@schreyoegg.de 


\section{Die verschiedenen Epochen des Werks von Moreno mit ihren jeweiligen Schwerpunkten}

Der breite, aber vielfach eher implizite Bezug auf Morenos Ansätze ist letztlich einem Lebenswerk geschuldet, das sich keineswegs so einfach und flüssig erschließt. Es manifestiert sich in unterschiedlichen Lebensstadien des Autors oft eher durch poetische Texte als durch klar und eindeutig formulierte Ausführungen, weshalb Morenos Werk heute eher durch Texte der Sekundärliteratur verbreitet wird. Und vielfach erschließt es sich dem Anwender erst durch das eigene Erleben. Das Moreno'sche Oeuvre lässt sich drei Stadien zuordnen, die mit dem Lebenslauf des Autors korrespondieren. Sie sind für uns hier gleichermaßen relevant und repräsentieren auch unterschiedliche rollentheoretische Positionen (Petzold 1982, 1984):

Jacob Levy Moreno wurde 1889 in Bukarest geboren und wuchs als ältestes Kind einer jüdischen Kaufmannsfamilie in Wien auf. Der Name „Moreno“ bedeutet im Hebräischen „unser Meister“. Er studierte Medizin und wurde Psychiater. Schon als Kind inszenierte er mit seinen Geschwistern kleine Theaterstücke. Im Verlauf seines Studiums kam er in Berührung mit Positionen des Expressionismus, einer Geisteshaltung, die sich gegen die Vereinnahmung des Menschen durch die Technik bzw. durch die Industrialisierung wandte. Als Protagonist dieser Bewegung fungierte er früh als Herausgeber einer expressionistischen Zeitschrift, des „Daimon“. Expressionistisches Gedankengut regte ihn auch zu neuartigen Arbeitsformen mit seinen Patienten an. Er animierte sie, im Sinne von Katharsis alles das, was sie innerlich bewegte, auf einer Bühne auszuagieren, also sich selbst zum Ausdruck zu bringen. Dabei verstand er Katharsis nicht, wie von Freud und Breuer ursprünglich praktiziert, als einfaches Ausagieren. Er beabsichtigte vielmehr ähnlich dem antiken Drama eine Läuterung der Gefühle, die dem Menschen ein neues Gleichgewicht ermöglichen solle. Moreno knüpfte damit allerdings auch an ältere Psychiater an, die schon an der Schwelle zum 19. Jahrhundert mit dem dramatischen Spiel in der Arbeit mit Geisteskranken experimentiert hatten (Petzold 1984, S. 114). Zeitgleich mit Morenos Experimenten entwickelte Stanislawskij seine berühmte Theatertheorie, wonach Schauspieler nicht einfach nur die Rolle eines Stückes auf der Bühne zu personifizieren hatten, sondern mit der jeweiligen literarischen Rolle maximal verschmelzen sollten. Schon aus dieser Epoche schöpfte Moreno Vorstellungen über eine kreative Lebens- und Arbeitsgestaltung, über Sinn und Verantwortung von Menschen. Hier erkannte er auch schon den Wert von Spontaneität, Kreativität und Begegnung, die den Kern seiner Anthropologie bilden.

Von 1915 bis 1917 wurde Moreno als medizinischer Betreuer des Flüchtlingslagers in Mitterndorf engagiert, wo er sich erstmalig mit soziometrischen Phänomenen befasste. Bei den Bewohnern handelte es sich um zwangsevakuierte Italiener vor allem aus dem Trentino. 1918-1925 war er Werks- und Gemeindearzt in Vöslau. 1925 schließlich, also vor der Nazizeit, emigrierte er nach New York, wo er zunächst als Gefängnispsychiater tätig wurde. Die Leitung eines dortigen Mädchenheims hatte auch von Morenos Soziometrie gehört und bat ihn, in ihrer Einrichtung die permanenten Streitigkeiten unter den Mädchen zu regulieren. $\mathrm{Zu}$ dieser Zeit beschäftigten sich etliche Autoren mit menschlichem Lernen bzw. die ersten Lerntheorien wurden erforscht. Und so integrierte auch Moreno in sein Schaffen behavioristische Aspekte, wodurch er seine Soziometrie umfas- 
sender ausbaute. Vor allem mit Hilfe seiner berühmt-berüchtigten Befragungssoziometrie gelang es ihm, wohl mehr Ruhe in das Mädchenheim zu bringen. Die Mädchen wurden gefragt: „Mit wem möchtest du lernen, mit wem möchtest du wohnen?“ usw., damit die Heimsituation entsprechend den Befragungsergebnissen umgestaltet werden könnte. Von nun an diente das „Vermessen von Beziehungen“ der sozialpsychologischen Forschung; die Soziometrie diente aber auch als methodisches Gerüst für die pädagogische und therapeutische Arbeit. In den USA gab Moreno ab 1936 die Zeitschrift „Sociometric Review“ heraus. In diesem Zusammenhang beschäftigte er sich auch als einer der ersten mit Gruppenpsychotherapie und mit Aktionsforschung.

Erst in seinem Spätwerk entwickelte Moreno das Psychodrama als Verfahren mit seinen verschiedenen agogischen Varianten und seinem breiten Methodenuniversum. Dabei handelte es sich im Wesentlichen um eine Arbeit mit Gruppen. Als psychodramatische Arbeitsformen lassen sich etliche Arrangements nennen: 1) das Stehgreifspiel, bei dem Spieler aus dem Stand ohne Vorlage zu einem Thema eine Szene gestalten, 2) das Rollenspiel, bei dem Spieler eine Rollenvorlage ausgestalten, 3) das Soziodrama, bei dem eine Gruppe von Personen eine soziale Konstellation mit grob vorgegebenen Rollenangaben spielt, und 4) das Psychodrama, das allerdings auch wieder in verschiedenen Varianten realisiert werden kann. Am bekanntesten ist das klassische, „Protagonisten-zentrierte Psychodrama“, bei dem eine zumeist traumatische Erfahrung einer Person mit Rollenspielpartnern aus der Gruppe inszeniert wird. Daneben existiert das „themen-zentrierte Psychodrama“, bei dem Teilnehmer Lebenssequenzen zu einem bestimmten Thema, z.B. ,als ich einmal eifersüchtig war“ usw., inszenieren, und das „Gruppen-gerichtete Psychodrama“, bei dem Fragestellungen bearbeitet werden, die sich aus einer aktuellen Gruppensituation ergeben (Petzold 1979). Zur Realisierung dieser verschiedenen Arbeitsformen nutzt man etliche methodische Maßnahmen wie den Rollentausch, d.h. Personen tauschen im Spiel ihre Rollen; den Rollenwechsel, d.h. eine Person wechselt in verschiedene eigene Rollen, z. B. Vater und Geschäftsmann; das Spiegeln, d. h. Mitspieler führen einem Protagonisten sein eigenes Verhalten vor, und das Doppeln, d.h. der Leiter oder Mitspieler „flüstern“ dem Protagonisten Gedanken ein, die der Protagonist sich vielleicht noch scheut auszusprechen. Zentrale Methoden sind Awareness-Übungen und Imaginationen. Awareness begreift man im Deutschen ähnlich wie „Achtsamkeit“. Das ist die maximale Konzentration auf innere oder äußere Reize und ihre Wahrnehmung mit allen Sinnen. Imagination heißt, Erfahrungen früherer oder zukünftiger Ereignisse sich vorzustellen und gegenwärtig zu setzen. Moreno hatte postuliert, dass sich der Mensch vom Tier vor allem darin unterscheidet, dass er durch Imagination die Zukunft wie die Vergangenheit gegenwärtig setzen kann. Das sei auch die ganz besondere Freiheit des Menschen. So kommt im Psychodrama neben Auseinandersetzungen mit der Vergangenheit auch den Zukunftsimaginationen eine große Bedeutung zu.

\section{Die verschiedenen Rollenkonzepte von Moreno}

Das gesamte Schaffen Morenos ist vom Früh- bis zum Spätwerk von drei unterschiedlichen Rollenkonzepten durchzogen. Petzold (1982) bezeichnet das erste als ,aktionale“ 
Rollentheorie, das zweite als ,,kategoriale“ und das dritte als reife Form einer Rollentheorie, weil es Moreno in diesem Stadium gelingt, die beiden ersten zu integrieren.

In seinem Frühwerk, das im Wesentlichen durch den Expressionismus geprägt ist, propagiert Moreno anlässlich seiner Theaterexperimente den „Spielmächtigen“, aus dem seine innere Welt im Spiel gleichsam herausquillt. Das Gegenstück ist der brave Schauspieler, der gewissermaßen perfektionistisch eine literarische Vorlage als „Rollenkonserve" unabhängig von seinem eigenen Sosein zu realisieren sucht. Schon bei seinen ersten psychiatrischen Theaterversuchen wendet sich Moreno - ähnlich wie ehedem Dahrendorf (1959, gegen Parsons 1951) - gegen die Sichtweise, Menschen hätten als Sozialpartner immer nur soziale Erwartungen anderer in ihrem Handeln zu realisieren. Er will vielmehr Menschen animieren, sich spontan, eigenständig und kreativ aus „Rollenkonserven“ zu befreien. Diese expressionistische Sicht von Rolle lässt sich geradezu als „Antirollentheorie“ bezeichnen (Petzold 1984).

Die kategoriale Rollentheorie der mittleren Schaffensperiode wird eingeleitet durch soziometrische Untersuchungen. Hier versteht Moreno die Bedeutung sozialer Rollen ähnlich dem Kulturanthropologen Linton (1947) als strukturelle Phänomene, die unabhängig von einem jeweiligen Menschen in einem gesellschaftlichen Ensemble schon traditionell existieren. „Vater“, „Mutter“, „Arzt“, ,Soldat“ usw. begreift er nun als gesellschaftliche Konserven, die in ihrer spezifischen Position und in ihrem gesellschaftlichen Status vorgegeben sind und dann vom Einzelnen wie selbstverständlich als Rolle verkörpert werden. Im Gegensatz aber zu Linton (und Parsons) stellt er diese kategorialen Rollen nicht als das Gesamt der Verhaltenserwartungen dar, sondern als das Gesamt der Verhaltensweisen, die aus einem komplexen Sozialprozess resultieren. Dabei unterscheidet Moreno (1934) noch drei Kategorien:

- Soziodramatische Rollen als Verkörperung von Handlungsmustern, in denen sich kulturell vorgegebene Rollenvorstellungen manifestieren, also die gesellschaftlich vermittelte Form, Vater, Mutter, Vorgesetzter, Arzt usw. zu sein.

- Psychodramatische Rollen als Verkörperung von Handlungsmustern, mit denen das Individuum seine eigene soziale Realität interpretiert. Das ist dann seine je spezifische Weise, Vater, Mutter oder Vorgesetzter zu sein.

- Psychosomatische Rollen als Verkörperung vitaler Grundmuster, wie der „Esser“, der „Schläfer" usw.

Bei psycho- und soziodramatischen Rollen unterlegt Moreno immer ein komplementäres Verhältnis von Rollenpartnern. Die Rolle der Mutter etwa konstituiert sich erst durch die Komplementärrolle des Kindes usw. Wie Mead (1973) sieht aber auch Moreno in dieser Phase Rollen immer situationsbezogen und als das Ergebnis sozialer Handlungsprozesse, in denen auch eine symbolische Vermittlung erfolgt (Petzold 1982). Anders als Mead will er aber nicht das ihm zu passivistisch erscheinende Verständnis von „role taking“ übernehmen, sondern spricht im Sinne seines aktionsorientierten Ansatzes von ,role playing“ (Moreno 1934). In dieser Schaffensperiode trainiert er seine Klienten, ihre psycho- und soziodramatischen Rollen entsprechend ihren eigenen schöpferischen Möglichkeiten und ihren psychosomatischen Rollen - auszugestalten.

Die rollentheoretische Position des Spätwerks: In seinem Spätwerk, in dem das therapeutische Handlungsmodell weiter verdichtet wird, begreift Moreno kategoriale Rollen 
zwar als individuell und sozial stabilisierend. Im Anschluss an seine frühen ,aktionalen“ Rollenvorstellungen postuliert er aber, dass sie immer wieder der Neugestaltung bedürfen, um nicht zur beengenden „Konserve“ zu erstarren (Buer 2010). Dies versucht er mit Hilfe seines Handlungsmodells, dem „Psychodrama“. Wie bei Plessner (1953) konzeptionell vorgesehen, soll der Mensch durch das Einnehmen „exzentrischer Positionen“, was Moreno durch seine Rollenspielmethodik zu realisieren sucht, immer wieder aktionale und kategoriale Rollenanteile integrieren.

\section{Der philosophische Hintergrund Morenos}

Morenos Philosophie ist für sein Werk von besonderer Bedeutung (Leutz 1974; Buer 1989, 1999, 2010; Petzold 1979, 1984 u. a.). Er hat im Laufe seines Lebens eine Vielzahl von Strömungen zu integrieren versucht. Zuerst hat er aus der jüdischen Tradition geschöpft, sodann aus dem Expressionismus. Später berief er sich auf Kierkegaard und Nietzsche, auf Darwin und Bergson. Das ergab bei ihm (Moreno 1973) eine spezifische Kosmologie und Anthropologie. Moreno postuliert vier Universalia: Kosmos, Zeit, Raum und Realität. Der Kosmos befinde sich in ständiger Bewegung; Zeit gewinne nur im Augenblick existenzielle Bedeutung, der Mensch sei immer nur in seinem jeweiligen Kontext zu verstehen, und die Realität könne der imaginationsfähige Mensch immer durch eine „surplus Realität“ in die Zukunft und in die Vergangenheit erweitern.

In Morenos Anthropologie stehen Begegnung, Sozialität, Spontaneität und Kreativität im Vordergrund. Gesundheit resultiere dann aus der maximalen Entfaltung dieser Aspekte. Neben Postulaten der medizinischen Ethik propagierte Moreno Engagement für das Überleben der Menschheit und des gesamten Kosmos. In seinen gesellschaftstheoretischen Utopien spielt die Soziometrie eine ganz besondere Rolle. Er postuliert, dass jede gesellschaftliche Entwicklung aus einem Stadium der politischen Kreativität, wie man sie nach Revolutionen vorfindet, in eine Epoche der formalisierten Verfestigung mündet. Diese muss dann durch gezielte soziometrische Arbeit immer wieder aufgelöst werden.

Im vorliegenden Heft von OSC finden sich nun einige Beiträge, die auf unterschiedliche Aspekte des Schaffens von Moreno Bezug nehmen: Im ersten Aufsatz beschäftigt uns Markus Schmitz mit dem Ertrag der „Soziometrie fürs Coaching“. Claus Rothenbücher stellt „die Organisationsaufstellung im Konfliktmanagement“ vor, die methodisch ebenfalls auf Morenos Werk zurückreicht. Anschließend präsentiert uns Michael Döller seinen Beitrag über „Begegnung als Maxime und Intervention im Coaching“, womit der Autor auf Morenos anthropologisches Konzept der Begegnung rekurriert. Der vierte Beitrag von Martin Neumann ist einer kreativen Arbeitsform gewidmet, dem „Einsatz literarischer Texte im Coaching", was sich in Morenos Intentionen zum kreativen Arbeiten gut integrieren lässt. Im Diskurs von Ferdinand Buer blitzen noch einmal anthropologische Reminiszenzen an Moreno auf. Hier antwortet der Autor auf einen Beitrag aus Heft 4/2011 von den Heidelberger Systemikern Mirko Zwack, Audris Muraitis und Jochen Schweitzer-Rothers, indem er deren normative Positionen mit denen des Psychodramas konfrontiert. 


\section{Literatur}

Buer, F. (1989). Morenos therapeutische Philosophie. Eine Einführung in ihre kultur- und ideengeschichtliche Kontexte. In F. Buer (Hrsg.), Morenos therapeutische Philosophie (S.9-42). Opladen: Leske + Budrich.

Buer, F. (1999). Lehrbuch der Supervision. Münster: Votum.

Buer, F. (2010). Psychodrama und Gesellschaft. Wege zur sozialen Erneuerung von unten. Wiesbaden: VS Verlag für Sozialwissenschaften.

Buer, F., \& Schmidt-Lellek, C. (2008). Life-Coaching. Über Sinn, Glück und Verantwortung in der Arbeit. Göttingen: Vandenhoeck \& Ruprecht.

Dahrendorf, R. (1959). Homo Sociologicus. Köln: Westdeutscher.

Leutz, G. (1974). Psychodrama. Berlin u. a.: Springer.

Linton, R. (1947). Role and status. In E. H. Newcomb \& E. L. Hartley (Hrsg.), Readings in social psychology. New York: Allyn \& Bacon.

Mead, G. H. (1973). Geist, Identität und Gesellschaft. Frankfurt a. M.: Suhrkamp.

Moreno, J. L. (1934). Who shall survive? A new approach to the problem of human interrelations. Washington: Nervous and Mental Disease Publ.

Moreno, J. L. (1973). Gruppenpsychotherapie und Psychodrama (2. Aufl.). Stuttgart: Thieme.

Neumann-Wirsig, H. (2009). Supervisions-tools. Bonn: ManagerSeminare.

Parsons, T. (1951). The social system. Glencoe: Routledge.

Petzold, H. (1979). Psychodrama-Therapie. Paderborn: Junfermann.

Petzold, H. (1982). Die sozialpsychiatrische Rollentheorie J. L. Morenos und seiner Schule. In H. Petzold \& U. Matthias (Hrsg.), Rollenentwicklung und Identität. Paderborn: Junfermann.

Petzold, H. (1984). Die ganze Welt ist eine Bühne. Das Psychodrama als Methode der klinischen Psychotherapie. In H. Petzold (Hrsg.), Wege zum Menschen I. Paderborn: Junfermann.

Plessner, H. (1953). Zwischen Philosophie und Gesellschaft. Bern: Francke.

Rauen, C. (Hrsg.). (2004). Coaching-tools. Bonn: ManagerSeminare.

Rauen, C. (Hrsg.). (2007). Coaching-tools II. Bonn: ManagerSeminare.

Schmidt-Lellek, C., \& Buer, F. (Hrsg.). (2011). Life-Coaching in der Praxis. Wie Coaches umfassend beraten. Göttingen: Vandenhoeck \& Ruprecht.

Witte, K. (2001). Wozu brauche ich auf geradem Weg einen Kompass? Gestaltung von Beziehungen in der psychodramatischen Supervision. OSC, 8(3), 213-234.

Witte, K. (2007). Freiwillige in Organisationen - störend oder bereichernd? OSC, 14(2), 131-143. 